\title{
Unscripted: A Cenotaph for Richard Feynman
}

ADAM MODESITT

Tulane University

Within highly complex computation-enabled assemblies, where can architects author composition? Within the procedural logic of individual components? Within procedural logic of the greater assembly? Or somewhere in-between? To these questions, this project proposes a simple answer: yes.

A speculative research project, the Cenotaph for Richard Feynman, explores methods for managing, organizing, and authoring complex geometric assemblies through drawing and modeling alone, without scripting, optimization, or other externalizations of compositional authorship. The project adhered to four self-imposed ground rules. First, no typing or coding. Interactive drawing and modeling only. Second, no splines or curvilinear surfaces, neither in the result nor in the design process. Rectangles and circles, cubes and spheres only. Third, no parametric, formal variation within individual components. Assemblies of standardized, discrete parts only. Forth, no fewer than million components. Massive assemblies only.

To follow these rules, a custom workflow in Dassault Systèmes' 3DEXPERIENCE platform was developed to execute the project, designed to manage assemblies of very large numbers of components using associative modeling. All project data and modeling occurred in a cloud-hosted database, without the use of files. The project was sponsored by Dassault Systèmes, a multinational software developer more closely associated with the automotive and aerospace industries than architecture, as means for exploring the use of software platforms under development for architectural production.

The Cenotaph for Richard Feynman was designed as a monument to honor the life and work of the physicist Richard Feynman. The project consists of steel trusswork organized around a system of interconnected distributed load paths as a structural analogue to Feynman's 'third way' path integral formulation of quantum mechanics. Sited in the industrial context of Newark Bay, New Jersey, the cenotaph is composed of over ten million standardized individual steel members that borrow the industrial tectonic of its context. Individual members aggregate into assemblies and sub-assemblies of trusses, each a unique configuration consisting of thousands of discrete components. What is automation's role in composition? A composer or a player in the orchestra? Perhaps it is neither, but instead an instrument capable of both noise and melody. It is up to us to decide the difference. 


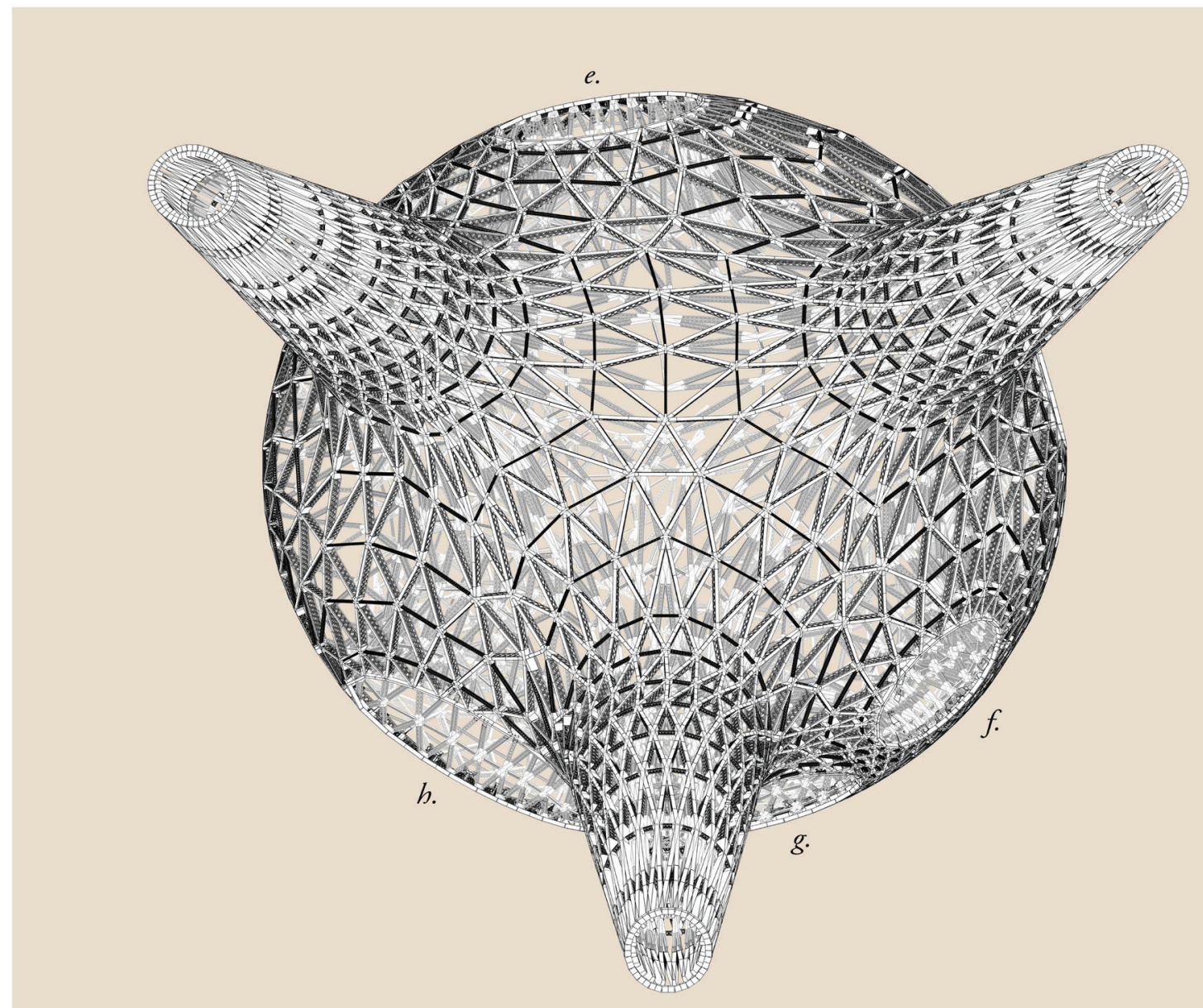

The Lower Caldera

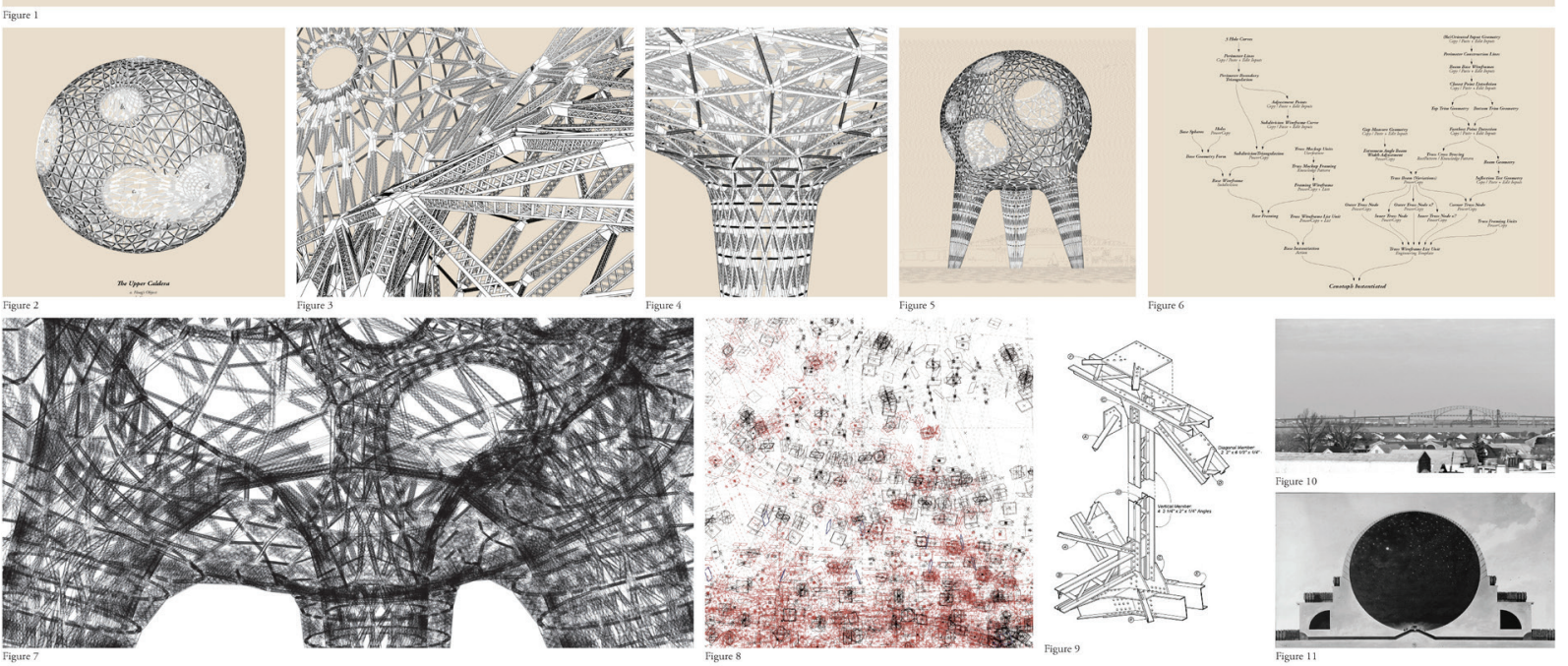

UNSCRIPTED A Cenotaph for Richard Feynman

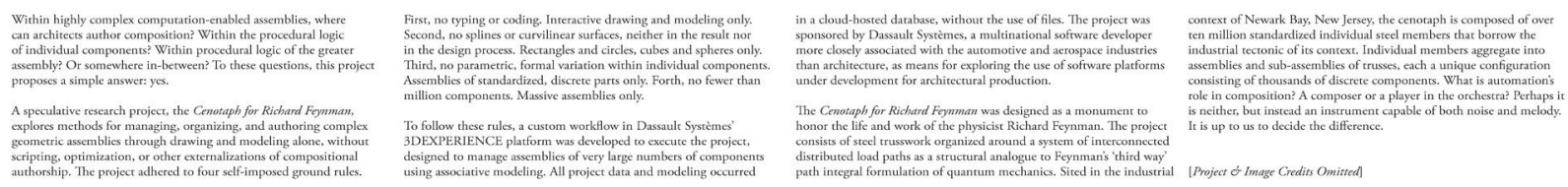

